\title{
Hidden Thermodynamic Information in Protein Amino Acid Mutation Tables
}

V. Sachdeva and J. C. Phillips

Dept. of Physics and Astronomy, Rutgers University, Piscataway, N. J., 08854

\begin{abstract}
We combine the standard 1992 20x20 substitution matrix based on block alignment, BLOSUM62, with the standard 1982 amino acid hydropathicity scale KD as well as the modern 2007 hydropathicity scale MZ, and compare the results. The 20-parameter KD and MZ hydropathicity scales have different thermodynamic character, corresponding to first- and second-order transitions. The KD and MZ comparisons show that the mutation rates reflect quantitative iteration of qualitative amino acid phobic and -philic binary $2 \times 10$ properties that define quaternary $4 \times 5$ subgroups (but not quinary $5 \times 4$ subgroups), with the modern MZ bioinformatic scale giving much better results. The quaternary 5-mer MZ 4x5 subgroups are called mutons (Mu5).
\end{abstract}

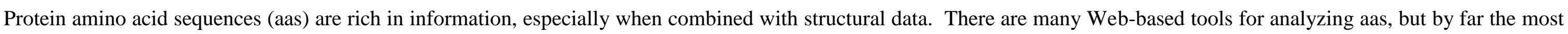

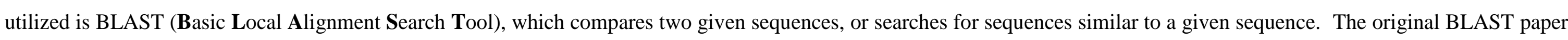

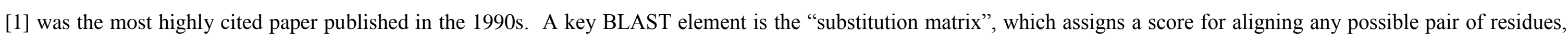

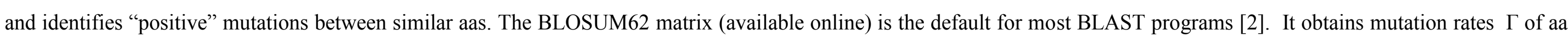
pairs from protein blocks (distantly related but conserved regions), which leads to accurate homological lists of functionally similar protein blocks.

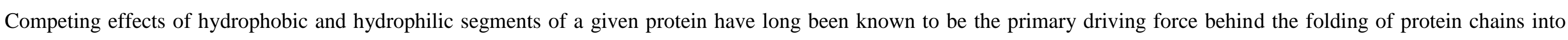

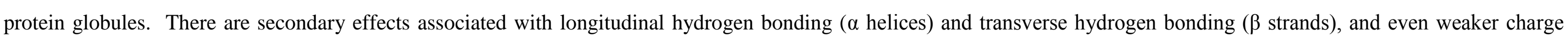
effects, but in most proteins the dominant physico-chemical factor in a kinetic property such as aggregation [3] is hydropathic interactions. 


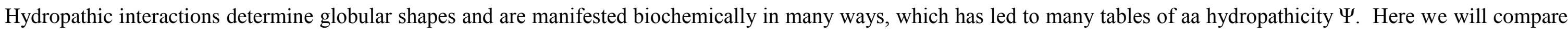

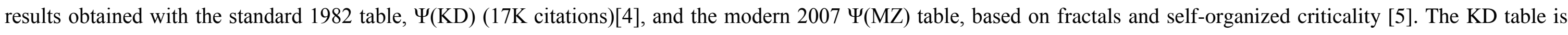

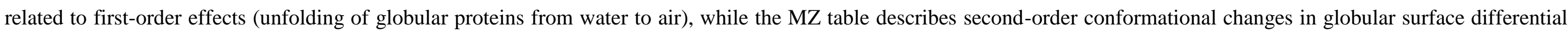
geometry [3]. Our analysis of the BLOSUM62 matrix will enable us to decide whether block homologies are primarily first- or second-order thermodynamically.

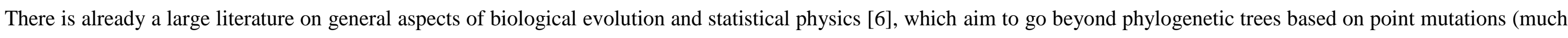

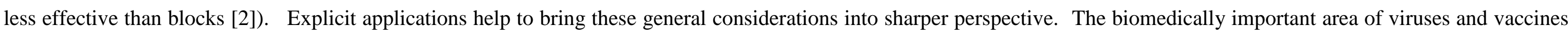
is best quantified using epitopes [7,8], which are similar to but still different from blocks, which are best suited to describing self-sustaining proteins [9].

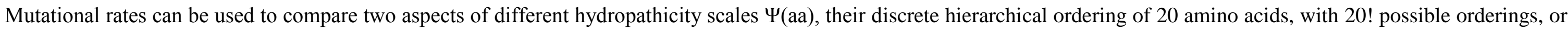

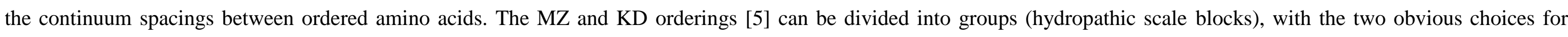

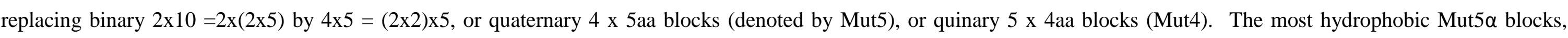

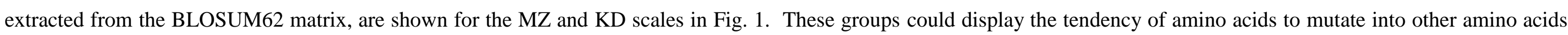
within their subgroup with similar hydropathicity.

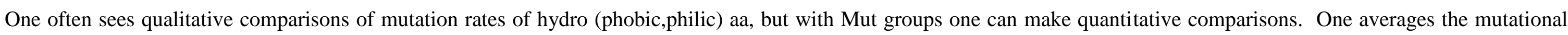

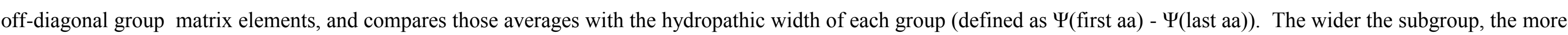

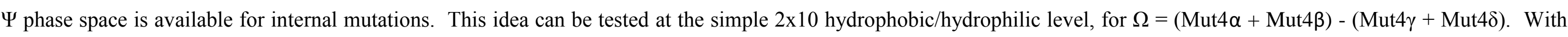
the MZ scale $\Omega=-1.2$ (as one might have expected, exposed hydrophilic aa mutate much more often than buried hydrophobic aa), but with the KD scale $\Omega=0.2$ (unsatisfactory).

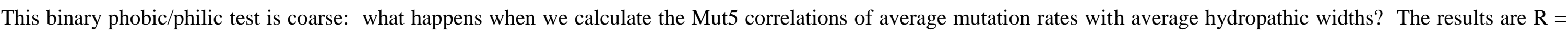

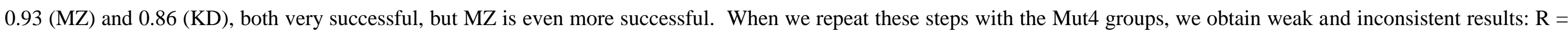

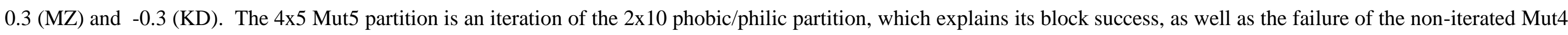
blocks. 


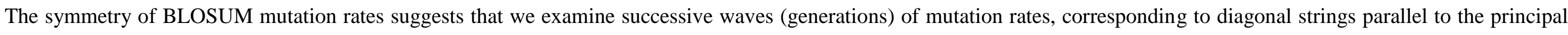

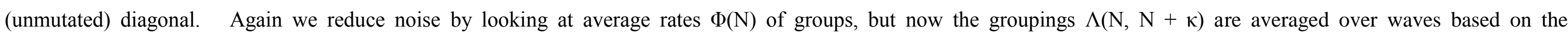

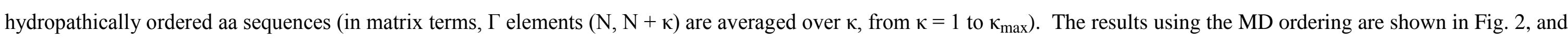
the KD ordering in Fig. 3, and discussed in those captions.

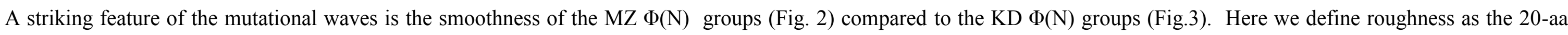

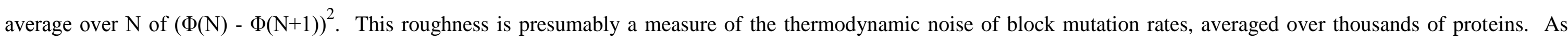

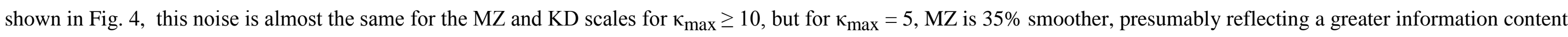

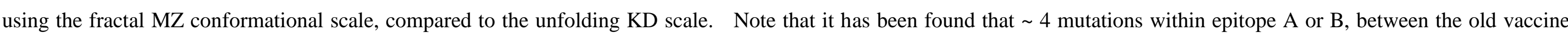

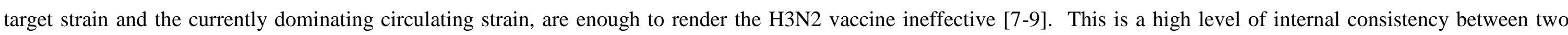
widely separated methods.

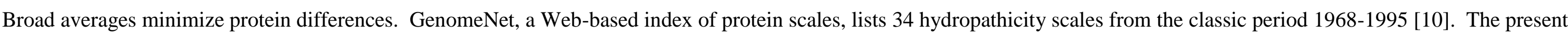

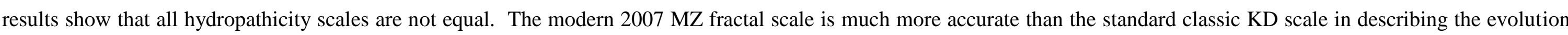

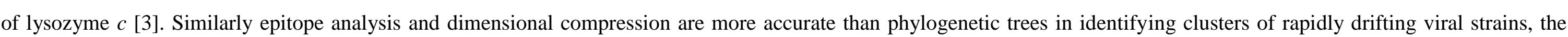
critical factor in engineering effective H3N2 vaccines [7-9] and arXiv 1510.00488.

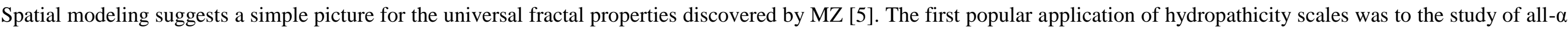

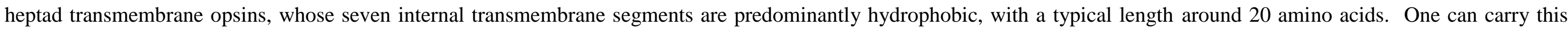

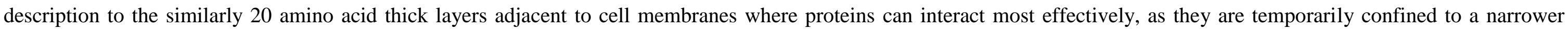

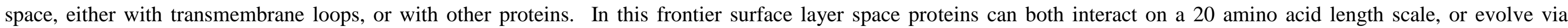
exchange of modular elements of $\sim 20$ amino acids. This picture is analogous to heterogeneous catalysis, with the membrane playing the part of catalytic substrate. 


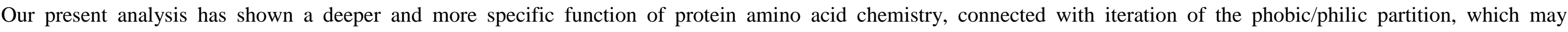

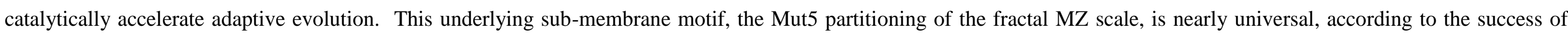

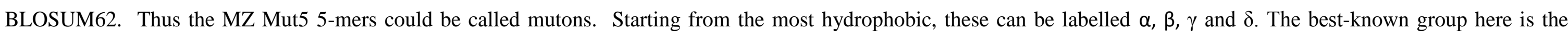

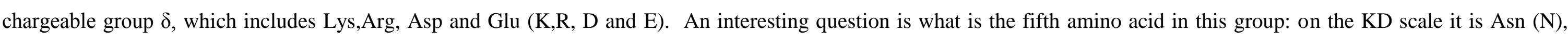
while on the MZ scale it is Ser (S). Elsewhere this question is discussed in detail, for analyzing the evolution of vaccine effectiveness for flu H3N2 [11,12]

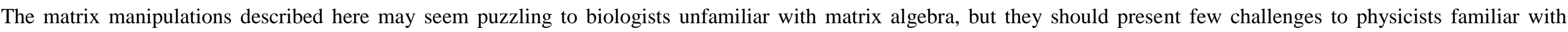

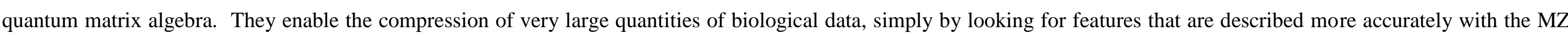

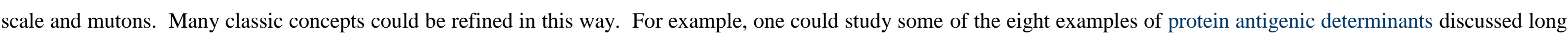

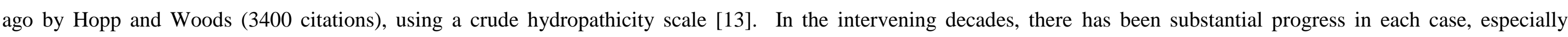

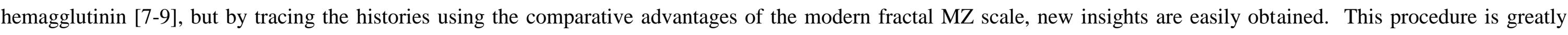
facilitated by using the Web of Science, a widely underutilized tool [14].

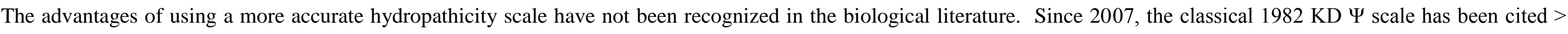

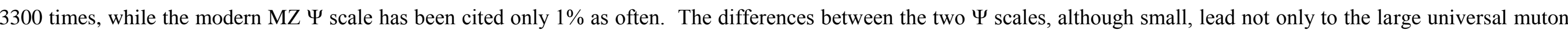

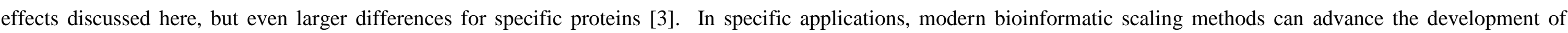

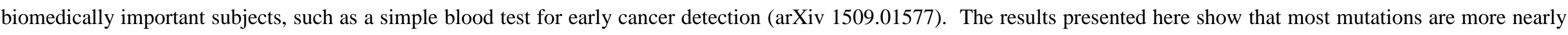

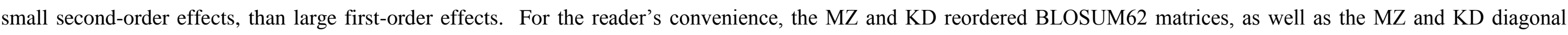
mutation wave tables, are attached to this paper at arXiv xxx. The MZ and KD $\Psi$ tables are in [15]. 


\begin{tabular}{|c|c|c|c|c|c|c|c|}
\hline \multirow[t]{2}{*}{-1} & -1 & -2 & -1 & 3 & 2 & 0 & -1 \\
\hline & 3 & -1 & 1 & & 1 & -1 & -1 \\
\hline MZ & & -1 & 1 & KD & & 0 & -1 \\
\hline$\alpha=$ CVI YM & & & -1 & $\alpha=\operatorname{IVLFC}$ & & & -2 \\
\hline
\end{tabular}

Fig. 1. Two sample Mut5 blocks, using either the MZ or KD hydropathicity scale. The integers represent logs of mutation rates, in (bit2)/2 units. Only the off-diagonal elements are shown, and the MZ $\alpha$ rows (columns) are labelled by VIYM (CVIY). For the MZ group $\alpha$, the hydropathic width is $\Psi(C)-\Psi(M)$. The KD block is similar, but with $\alpha=$ IVLFC. 


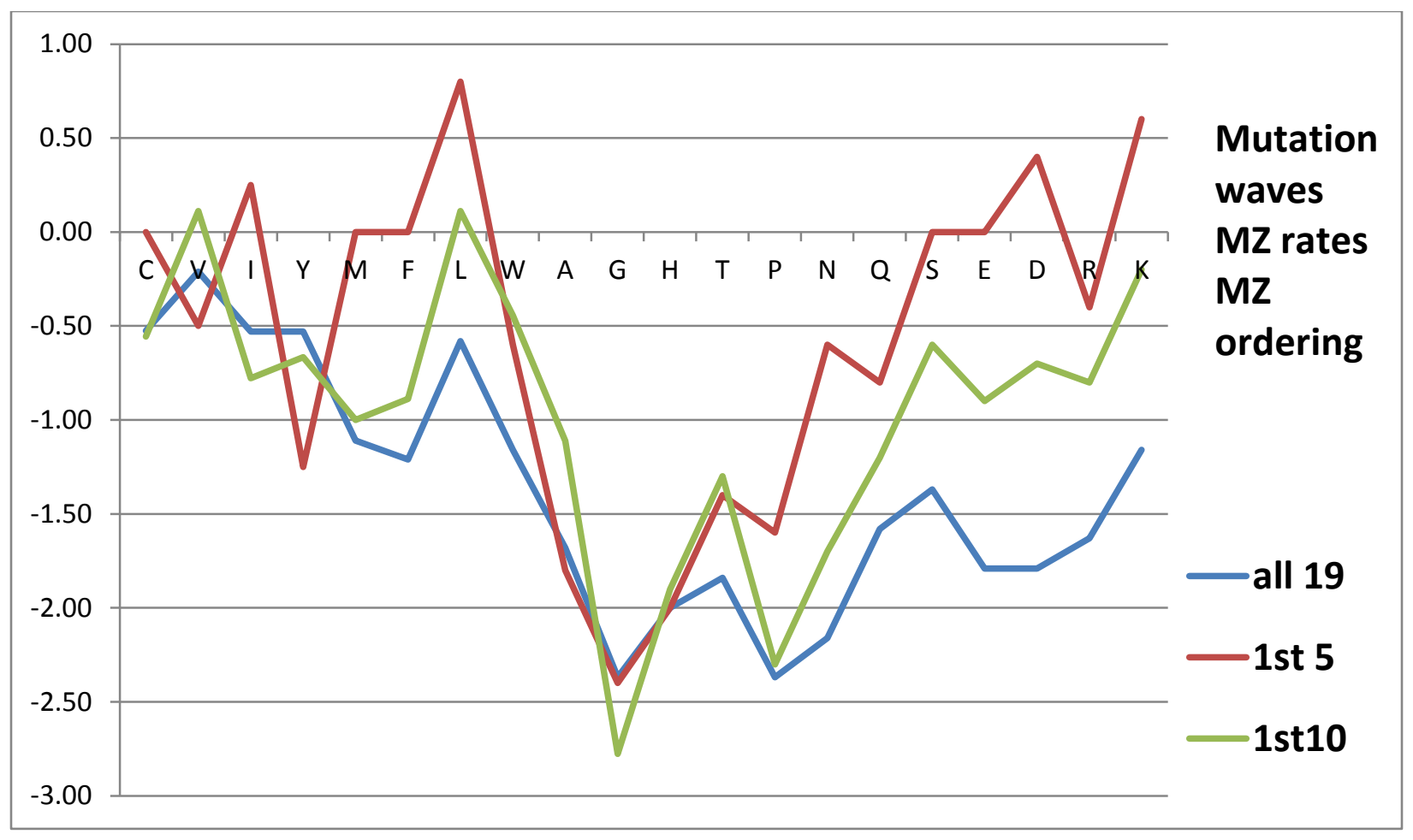

Fig. 2. When the MZ scale is used to construct mutation waves $\Lambda(\mathrm{N}, \mathrm{N}+\kappa)$, a strong dip in averaged wave rates $\Phi(\mathrm{N})$ is seen near the hydroneutral center aa. The hydrophobic and hydrophilic wings have higher mutational rates. Here $1^{\text {st }} 5$ means $\Phi(\mathrm{N})$ is obtained by averaging $\Lambda(\mathrm{N}, \mathrm{N}+\kappa)$ over $\kappa=1$ to 5 ,etc. The memory effects for larger $\kappa$ are large for the hydroneutral dip, and small for the hydrophilic wing. 


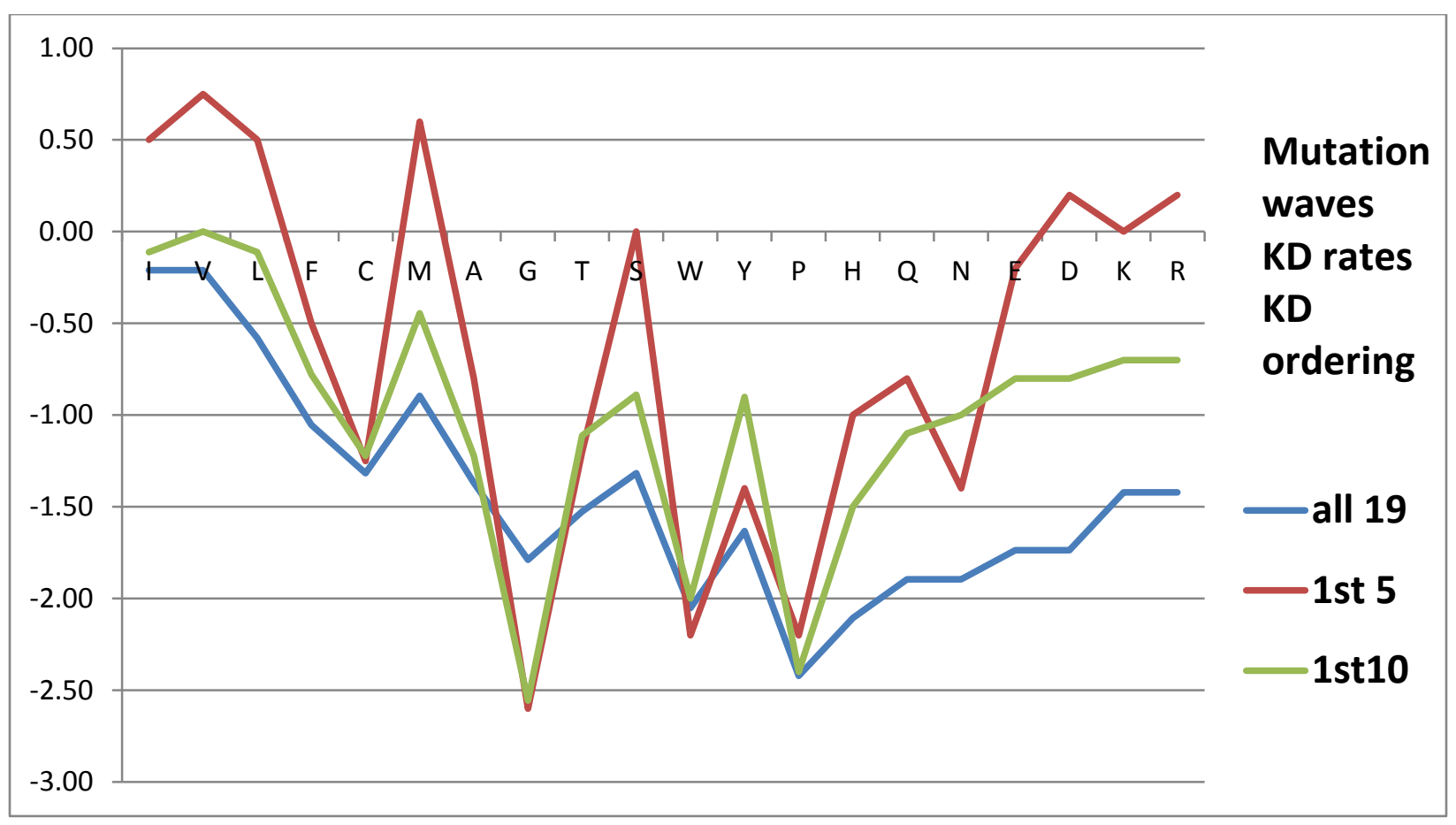

Fig. 3. This is similar to Fig. 2, except that here the KD hierarchy has been used to order the BLOSUM62 matrix [2]. Much of the simplicity of Fig. 2 has been lost, presumably because mutational substitutions tend to minimize MZ second-order conformational distortions, and do not involve the first-order unfolding effects described by the KD scale. 


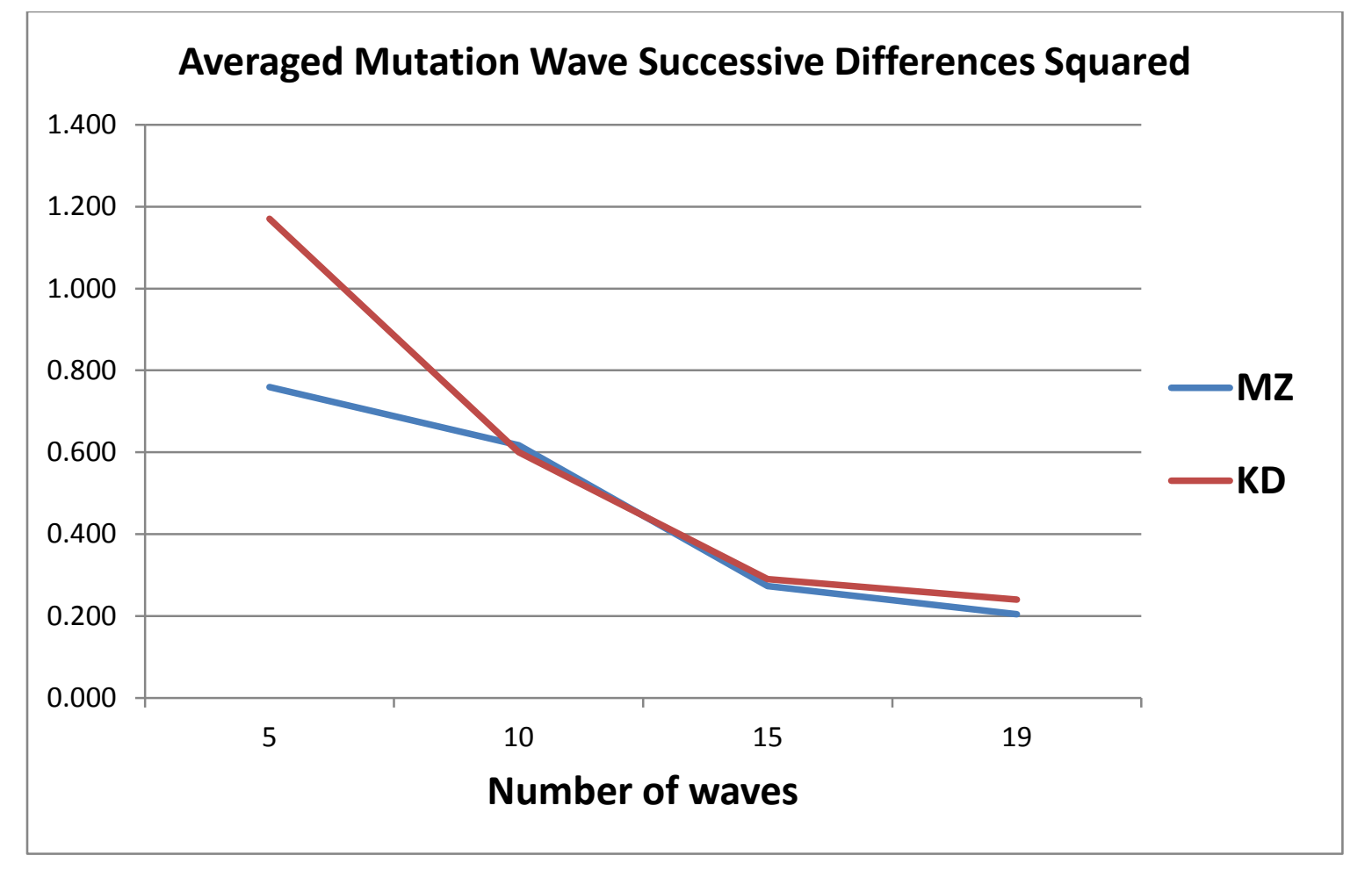

Fig. 4. Quantitative comparison of $\Phi(\mathrm{N})$ from Figs. 3 and 4 roughness $\Omega$, defined as average of $(\Phi(\mathrm{N})-\Phi(\mathrm{N}+1))^{2}$. The two orderings yield almost identical $\Omega$ for $\kappa_{\mathrm{max}} \geq 10$, but for $\kappa_{\max }=5$, the $\mathrm{MZ}$ ordering yields $35 \%$ smoother successive mutation rate waves. 


\section{References}

1. Altschul S, Gish W, Miller W, et al. (1990) Basic local alignment search tool. J. Mol. Bio. 215, $403-410$.

2. Henikoff S, Henikoff JG (1992) Amino-acid substitution matrices from protein blocks. Proc. Nat. Acad. Sci. (USA) 89, 10915-10919.

3. Phillips JC (2014) Fractals and Self-Organized Criticality in Proteins. Phys. A 415, 440-448.

4. Kyte J, Doolittle RF (1982) A simple method for displaying the hydropathic character of a protein. J. Mol. Biol. 157, $105-132$.

5. Moret MA, Zebende GF (2007) Amino acid hydrophobicity and accessible surface area. Phys. Rev. E 75, 011920.

6. Drossel B (2001) Biological evolution and statistical physics. Adv. Phys. 50, 209-295.

7. Munoz ET, Deem MW (2005) Epitope analysis for influenza vaccine design. Vaccine 23, 1144-1148.

8. Deem MW, Lee HY (2003) Sequence space localization in the immune system response to vaccination and disease. Phys. Rev. Lett. 91 , 068101.

9. Bogarad LD, Deem MW (1999) A hierarchical approach to protein molecular evolution. Proc. Nat. Acad. Sci. (USA) 96, 2591-2595.

10. Kawashima S, Pokarowski P, Pokarowska M, et al. (2008) AAindex: amino acid index database, progress report 2008. Nucl. Acids Res. 36, D202-D205.

11. Pan, K; Long, J; Sun, H; Tobin, GJ; Nara, PL; Deem, MW (2011) Selective pressure to increase charge in immunodominant epitopes of the H3 hemagglutinin influenza protein. J. Mol. Evol. 72, 90-103.

12. Phillips JC (2016) Evolution of Epitopic Chemistry of Flu H3N2 Strains.

13. Hopp TP, Woods KR (1981) Prediction of protein antigenic determinants from amino acid sequences. Proc. Nat. Acad. Sci. (USA) 78, 3824-3828.

14. Phillips JC (2015) Phase transitions in the web of science. Phys. A 428, 173-177.

15. Phillips JC (2009) Scaling and self-organized criticality in proteins: Lysozyme $c$. Phys. Rev. E 80, 051916. 
MZ Table

\begin{tabular}{|c|c|c|c|c|c|c|c|c|c|c|c|c|}
\hline w & A & G & $\mathrm{H}$ & $\mathrm{T}$ & $\mathrm{P}$ & $\mathrm{N}$ & $\mathrm{Q}$ & s & $\mathrm{E}$ & $\mathrm{D}$ & $\mathrm{R}$ & K \\
\hline-2 & -3 & -3 & -3 & -1 & -3 & -3 & -3 & -1 & -4 & -3 & -3 & -3 \\
\hline-3 & 0 & -3 & -3 & 0 & -2 & -3 & -2 & -2 & -2 & -3 & -3 & -2 \\
\hline-3 & -1 & -4 & -3 & -1 & -3 & -3 & -3 & -2 & -3 & -3 & -3 & -3 \\
\hline 2 & -2 & -3 & 2 & -2 & -3 & -2 & -1 & -2 & -2 & -3 & -2 & -2 \\
\hline-1 & -1 & -3 & -2 & -1 & -2 & -2 & 0 & -1 & -2 & -3 & -1 & -1 \\
\hline 1 & -2 & -3 & -1 & -2 & -4 & -3 & -3 & -2 & -3 & -3 & -3 & -3 \\
\hline-2 & -1 & -4 & -3 & -1 & -3 & -3 & -2 & -2 & -3 & -4 & -2 & -2 \\
\hline \multirow[t]{10}{*}{11} & -3 & -2 & -2 & -2 & -4 & -4 & -2 & -3 & -3 & -4 & -3 & -3 \\
\hline & 4 & 0 & -2 & 0 & -1 & -2 & -1 & 1 & -1 & -2 & -1 & -1 \\
\hline & & 6 & -2 & -2 & 0 & 0 & -2 & 0 & -2 & -1 & -2 & -2 \\
\hline & & & 8 & -2 & -2 & 1 & 0 & -1 & 0 & -1 & 0 & -1 \\
\hline & & & & 5 & -1 & 0 & -1 & 1 & -1 & -1 & -1 & -1 \\
\hline & & & & & 7 & -2 & -1 & -1 & -1 & -1 & -2 & -1 \\
\hline & & & & & & 6 & 0 & 1 & 0 & 1 & 0 & 0 \\
\hline & & & & & & & 5 & 0 & 2 & 0 & 1 & 1 \\
\hline & & & & & & & & 4 & 0 & 0 & -1 & 0 \\
\hline & & & & & & & & & 5 & 2 & 0 & 1 \\
\hline
\end{tabular}




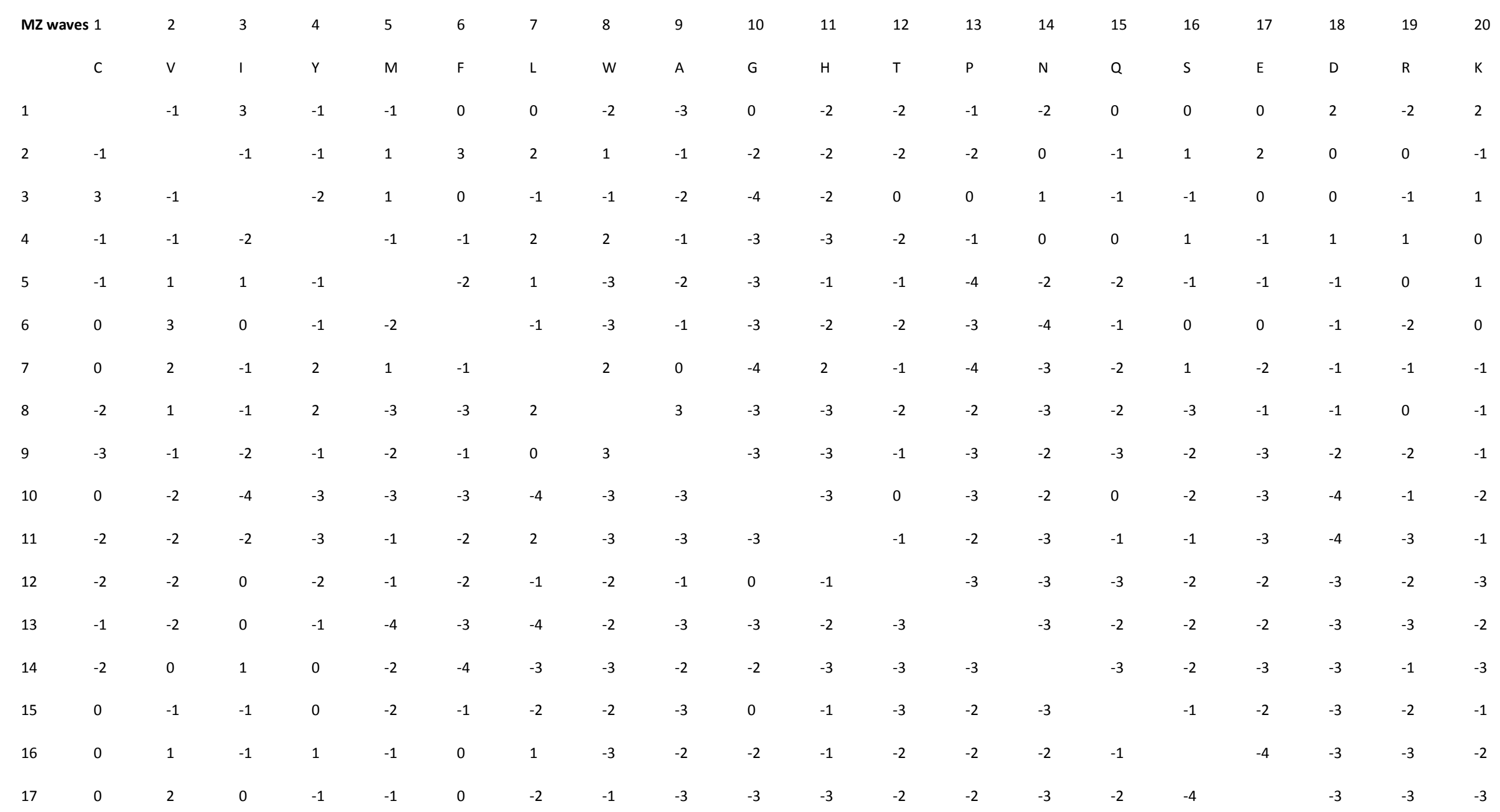




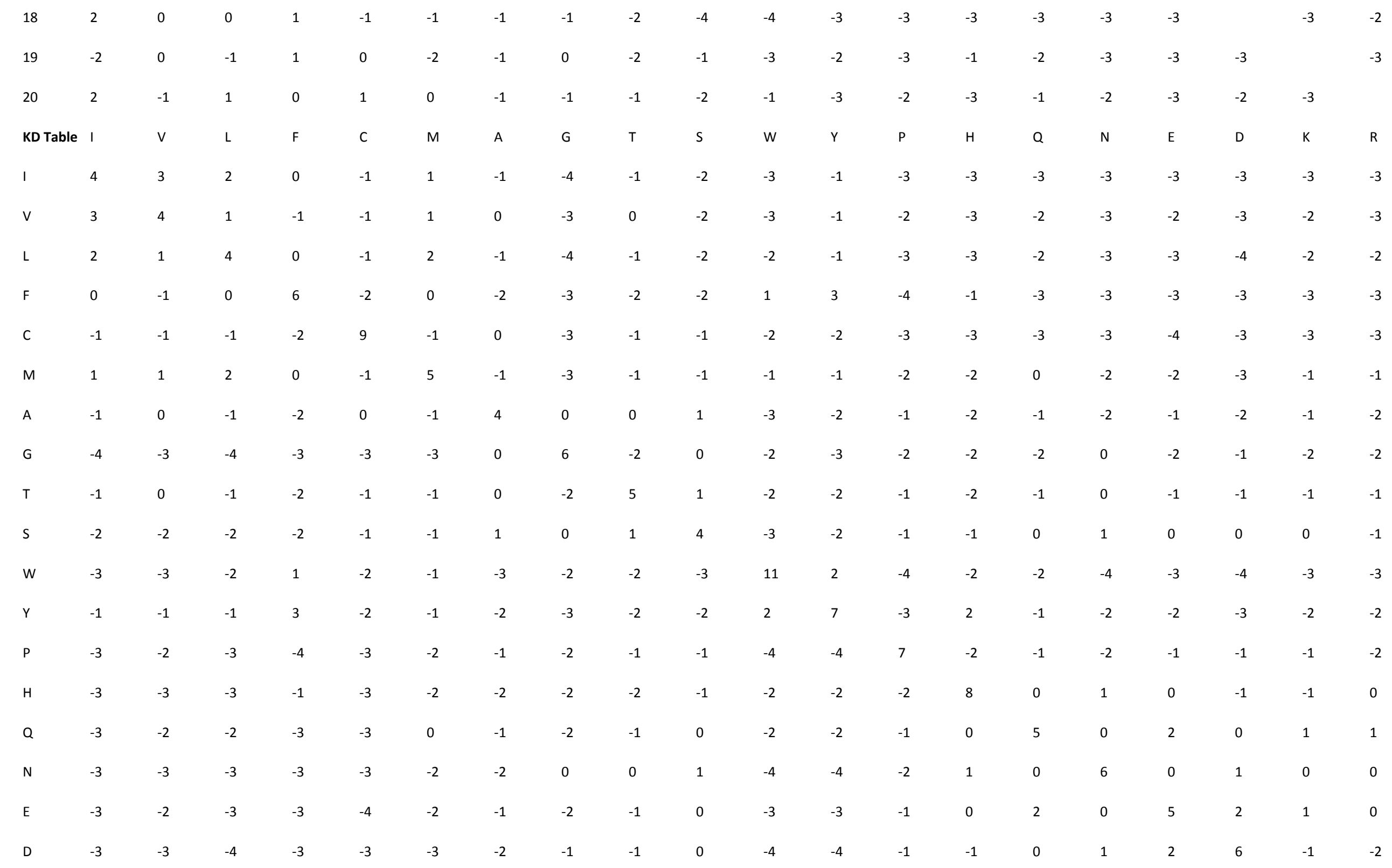




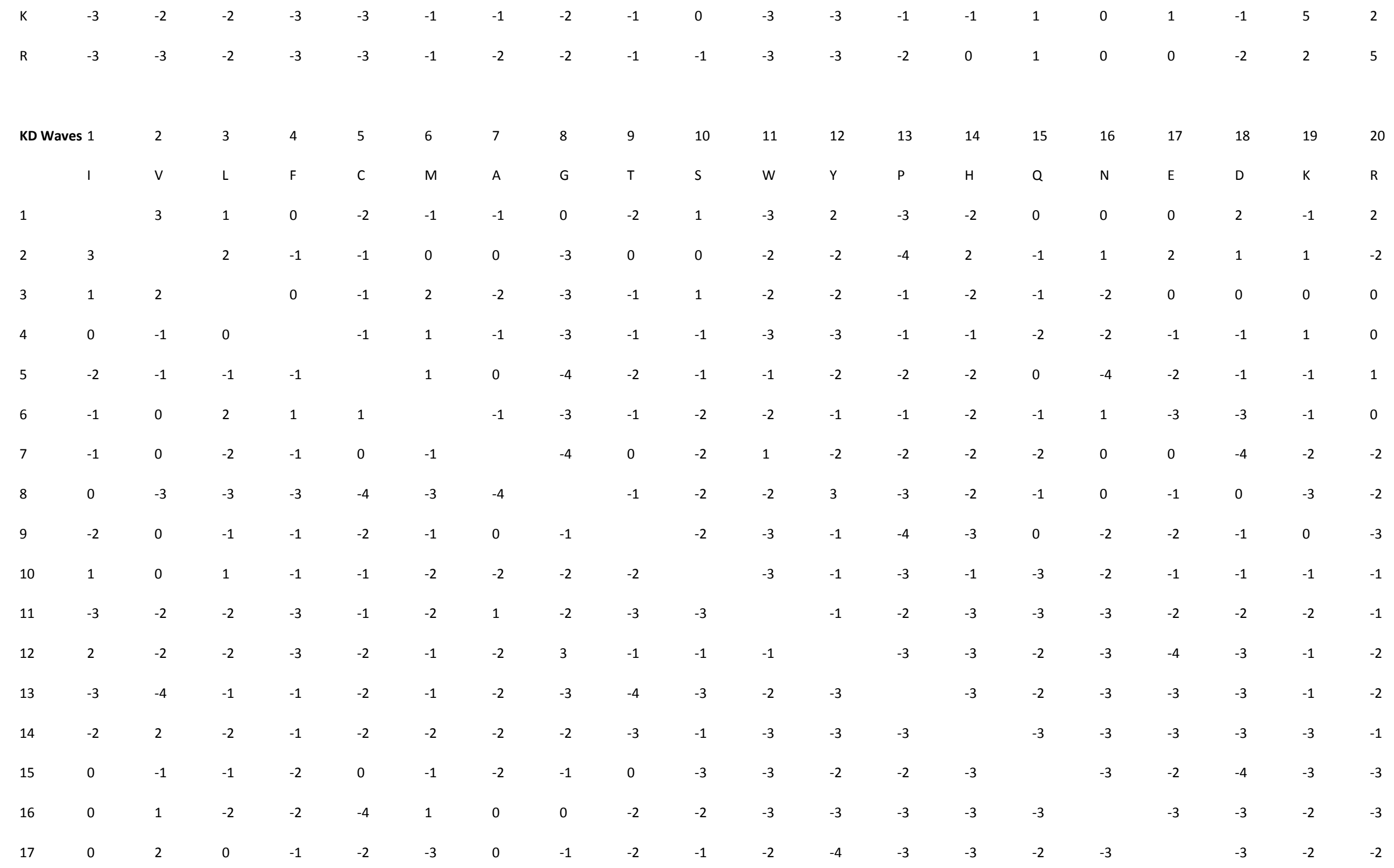




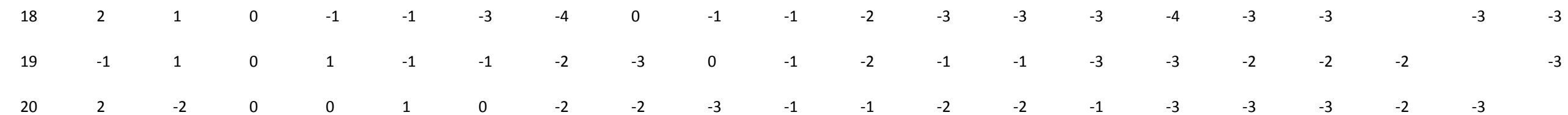

